\title{
Soft Matter and Biological Physics
}

(c) EDP Sciences / Società Italiana di Fisica / Springer-Verlag 2009

EPJE has a short but very successful history of publishing high-quality papers on experimental and theoretical soft matter physics. Starting with this issue, EPJE widens its scope to include all aspects of the study of biological systems by physical approaches. In the past, those areas of biological physics, such as biopolymers and membranes, which are allied most closely to the rest of soft matter physics, have been represented regularly in EPJE by excellent contributions. The opening of EPJE to cover the full spectrum of biological physics reflects the growing importance of this field, its strong conceptual and methodological links with soft matter physics, and gives biological physics a true home in the EPJ series.

EPJE aims to attract submissions in the broad area of biological physics, ranging from molecular machines on the nanoscale, to studies of the behaviours of multicellular systems and biological networks. Biological physics has in recent years undergone a rapid and dynamic development as many novel research avenues have been opened by new experimental techniques and by the rapid progress in molecular and cell biology. Because of their extraordinary complexity, biological systems pose enormous challenges. The systematic use of quantitative approaches as well as the combination of experiments and theory are still in their infancy in many areas of biology. Biological physics studies the new physics that emerges from novel insights into the properties and behaviours of living systems. Furthermore, it aims at developing new concepts and quantitative approaches for the study of biological phenomena. Approaches from soft matter physics and statistical physics, which have been at the core of EPJE, play a key role in this research.

We are looking forward to the broadening of contributions and encourage submissions that bring new subjects to EPJE.

R.A.L. Jones

F. Jülicher

G. Maret

Editors-in-Chief 